\title{
El impacto cultural del árbol de bálsamo en Chiltiupán, La Libertad, El Salvador
}

\section{The cultural impact of the balsam tree (Myroxylon balsamum var. pereirae) in Chiltiupán, La Libertad, El Salvador}

\author{
Julio César Martínez-Rivera' \\ Director de la Escuela de Antropología de la Utec \\ julio.martinez@utec.edu.sv \\ Julio Aníbal Blanco² \\ Rodrigo Antonio Colorado ${ }^{3}$ \\ Óscar Eduardo Guerra ${ }^{4}$ \\ Héctor David Antonio-Serrato 5 \\ Estudiantes Utec
}

Recibido: 02/03/2015 - Aceptado: 14/03/2015

\section{Resumen}

La producción del bálsamo ha sido clave para el desarrollo económico de la cordillera en la costa del occidente salvadoreño. Sin embargo, hay un componente de carácter histórico y cultural que parece bastante oculto y que el presente trabajo busca visibilizar al obtener información de los propios actores; como cultivadores, balsameros extractores, procesadores y habitantes del municipio de Chiltiupán en La Libertad, El Salvador.

La obtención de la información se produjo a través de un enfoque etnográfico, utilizando técnicas como la entrevista, la observación y los grupos focales junto con la búsqueda de información documental. El ordenamiento y análisis de la información produjo algunos datos y resultados, como el hecho de una mejor comprensión en torno a su historia prehispánica; la importancia extraeconómica del bálsamo en la región; las relaciones económicas y sociales que se producen en el entorno de

\section{Abstract}

The production of balsam has been a key point for the economic development of the Salvadorean mountain range in the western coast. Nonetheless, there exists a historical and cultural component which appears to be hidden; this work pursues its visibility by drawing information from the main people involved in it: growers, balsam extractors, processors and inhabitants of the municipality of Chiltiupán in La Libertad, El Salvador. In doing this work, an ethnographic approach was taken in terms of data collection; techniques such as the interview, observation and focus groups as well as documentary information were used. Information organization and analysis brought some data results such as the following: a better understanding about their prehispanic history, the extraeconomic relevance of balsam in the region, the social and economic relations that take place in the mountain range surroundings, the evolution of

Profesor investigador de la Universidad Tecnológica de El Salvador (Utec) y director de la Escuela de Antropología. Correo electrónico: julio.martinez@utec.edu.sv. Licenciado en Idiomas y estudiante de la carrera de Antropología. Profesor en Idioma Inglés de la Utec.

Estudiante de la Licenciatura en Antropología de la Utec.

Estudiante de la Licenciatura en Antropología de la Utec.

Estudiante de la Licenciatura en Antropología de la Utec. 
la cordillera; la evolución del mercado del producto; la importancia cultural de la transferencia generacional en la producción del bálsamo; las fuertes relaciones sociales en el marco de su producción y la distribución de los excedentes en la cadena de comercialización.

\section{Palabras clave}

Bálsamo, cultura, cordillera, economía. the market for this product, the cultural relevance of generational transfer in balsam production, the strong social relations within its production framework, and the surplus distribution in the marketing chain.

\section{Keywords}

Balsam [of El Salvador], culture, mountain range, economy.

\section{Introducción}

El bálsamo ha sido reconocido durante la historia colonial y moderna de El Salvador como uno de los principales productos agrícolas que han generado riqueza e identidad cultural. La costa salvadoreña, en particular la occidental, estaba cultivada de bálsamo (Browning, 1975) en su mayoría desde antes de la llegada de los españoles en 1524. Desde entonces, el producto ha sido crucial en la vida de los habitantes de la cordillera, que se ubica frente al océano Pacífico en el occidente del país, tanto en términos económicos como socioculturales.

Los abordajes de la temática previa del bálsamo se han enfocado en aspectos de carácter productivo agrícola (Castaneda Langlius, 1999), económicos (Fuentes, 1993), comercializadores (Anliker Palomo, 1981) y técnicas de emprendimiento (GTZ.Deutsche Gesellschaft für Technische Zusammenarbeit, S.f).

En términos productivos y estrictamente comerciales, durante la Colonia, el bálsamo tuvo un destacado auge (Lara Martínez, 2007), de tal forma que el Reino de España buscó proteger de los piratas a dicho producto, señalándolo como proveniente del Perú, como una forma de engaño a los ladrones (Alvarado, 1996) que buscaban el producto para Ilevarlo a Europa subrepticiamente.

Actualmente, el bálsamo continúa ofreciendo ingresos a los pobladores de la cordillera costera de El Salvador, llamada ejemplarmente "Cordillera del bálsamo" (Junio) (Alcaldía municipal de Chiltiupán, junio 2004); y ciertamente ha generado identidad cultural en su derredor, tanta que municipios completos y la región en general tienen como fuente de ingresos y distintivo cultural al producto mencionado.
El presente estudio aborda los aspectos de cultivo, extracción, procesamiento y comercialización del producto, desvelando la raigambre cultural insertada en esos procesos. Y también ofrece nuevas ideas para continuar con la investigación acerca del producto y para efectuar, tanto análisis culturales como económicos, y tener una perspectiva de desarrollo regional en torno al producto, siempre que se aborden los grandes problemas en su producción, tales como la propiedad de la tierra y las cadenas de comercialización.

\section{Metodología}

La presente investigación está concebido desde un proceso cualitativo, con un enfoque descriptivo etnográfico, usando la técnica documental y otras, como la observación directa. La entrevista abierta a informantes claves, así como el trabajo con los grupos focales, nos ha permitido documentar las historias y relatos de los participantes en la vida del municipio de Chiltiupán.

Se usó un método de recolección de información, que integró todas esas técnicas en las visitas de campo; y a estas se agregó la revisión documental en torno a la historia, los procesos y la comercialización.

Dentro de las técnicas que se desarrollaron está la consulta referencial histórico-cultural en fuentes documentales. El equipo de investigación, a partir de varias reuniones, estableció los lugares que se deberían visitar en el municipio; y se realizó el inventario de las fuentes documentales de la localidad, de centros académicos, bibliotecas y sitios en la red mundial de información.

Se realizaron entrevistas abiertas a través de un formato previamente validado, para lo cual se establecieron informantes claves dentro del municipio, que gozan de 
reconocimiento por la población de acuerdo con su profesión, labor de la promoción cultural y su sensibilidad con respecto a temas culturales identitarios. Para elegirlos se utilizó el criterio de conocimiento y vivencias en torno al tema.

Se realizó la observación directa por medio de una bitácora diaria de observación (Diario de campo). En esta se registraron todas aquellas manifestaciones culturales, elementos infraestructurales tangibles e intangibles, tanto en las visitas a las habitaciones de los balsameros como en los espacios abiertos en el pueblo. Así, se eligió a pobladores que laboran en fincas del entorno, siendo que su experiencia es contribuyente al estudio.

Se desarrollaron grupos focales que buscaron obtener información colectiva en torno a la percepción de los habitantes sobre el impacto del bálsamo en su vida y cultura.

\section{El bálsamo desde la historia y la literatura}

El abordaje de los elementos identitarios de El Salvador no solamente abarca los símbolos tradicionales patrios de todos conocidos (Angeloni, 2012). Hay que tener en cuenta que existen elementos naturales autóctonos que han sido exaltados para contribuir con la conformación de nuestra identidad nacional. Es decir, que el torogoz, el maquilishuat, y especialmente el bálsamo conforman esa identidad salvadoreña a partir de principios del siglo XX.

Junto con el cacao, el bálsamo constituye la primera reorganización agrícola, ya que sus propiedades medicinales y aromáticas eran conocidas antes de la Conquista (Browning, 1975). La demanda del bálsamo se difundió después de la Conquista por su empleo en medicina y como base para perfumes, lo que era común en Nueva España y Europa, donde la bulas papales de 1562 y 1571, de la Iglesia, autorizaban su uso para la confección del crisma y declaraba sacrilegio dañar o destruir el árbol del bálsamo (Browning, 1975).

La relación de la Iglesia con el bálsamo también es destacada por Lara (2007), quien enfatiza que "en la Colonia se oculta su procedencia bajo el título de Bálsamo del Perú". Esta iniciativa histórica se gestó debido a que era necesario esconder el origen, tanto de su plantación como de sus productos, de piratas depredadores que asechaban las zonas costeras de nuestro país en tiempos posteriores a la Conquista.

Para comenzar, es necesario trazar una línea en el tiempo para identificar la información histórica disponible que justifique la importancia de este árbol. En este sentido, uno de los más reconocidos escritores salvadoreños expresa que "el árbol del bálsamo es como el eucalipto, un árbol místico: el árbol sacerdote entre los del bosque (por qué no decir, el árbol santo)" (Salarrué, 1974). Esta contemplación mística destaca a este árbol en relación con otros que han sido considerados más comunes y populares en el contexto salvadoreño.

Al igual que el cacao, los españoles obtenían el bálsamo de los indígenas por explotación de la encomienda, ${ }^{6}$ compra o trueque. La recolección de la savia se convirtió en una actividad importante en un pequeño grupo de pueblos de los alrededores de Guaymango, al suroeste del distrito de Izalco; y esta región costera, que era la única de América que producía bálsamo, llegó a ser conocida como Cordillera del Bálsamo (Browning, 1975).

“En el postclásico, su asociación con el dios patrono de los mexicas Huitzilopochtli lo convierte en 'perfume de las princesas aztecas, de sacerdotes mayas, de Montezuma y de la Malinche' " (Lara Martínez, 2007). Se destaca que la complejidad de su valor sagrado y utilitario concede paulatinamente estatus sociales específicos donde las mujeres y líderes religiosos son los usuarios más simbólicos (Lara Martínez, 2007).

En 1984 ya se afirma que el árbol del bálsamo es "legítimamente de esta República" (Canales, 1982). La defensa de esta declaración está relacionada con el origen del 'bálsamo del Perú' y la confusa anticipación conceptual de considerar que el bálsamo de El Salvador es procedente del país sudamericano.

El Estado salvadoreño eligió como árboles nacionales de la República al bálsamo y al maquilishuat a través de Decreto Ejecutivo del 26 de junio de 1939 (Gobierno de El Salvador, 1939, pág. 1).

\footnotetext{
${ }^{6}$ La encomienda es una figura de propiedad en consignación que permitía al español contar con un grupo de indígenas para evangelizarlos y una hacienda. EL tamaño de la hacienda era concordante con el número de indígenas "encomendados", de tal forma que no solo se evangelizaban sino que también se explotaban.
} 
El impacto cultural del árbol de bálsamo en Chiltiupán, La

Libertad, El Salvador. Julio César Martínez-Rivera, Julio Blanco, Rodrigo Colorado, Óscar Guerra, Héctor Antonio, pp. 25-33.
Revista entorno, abril 2015, número 58.

ISSN: 2218-3345.
Este decreto abarcó varios territorios, pero singularmente los que ya tenían dentro de sus plantaciones al bálsamo, entre estos el municipio de Chiltiupán, que pertenece al departamento de La Libertad, y está limitado al norte por Jayaque, al sur por el océano Pacífico, al este por Teotepeque y Jicalapa y al oeste por Tamanique, esta situado a $750 \mathrm{msnm}$ y a $21.5 \mathrm{~km}$ al suroeste de la ciudad de Nueva San Salvador. (Alcaldía municipal de Chiltiupán, junio 2004). Chiltiupán está constituido por 11 cantones y 27 caseríos, distribuidos en el territorio del municipio (Recinos, 2008).

\section{Resultados y discusión}

El significado de la palabra cultura es polisémico, proviene del latín y se hace referencia al cultivo en proceso de mejora; se considera que aparece ligado al concepto de civilización (Hope Ponce, 2000). Esta concepción nos da la posibilidad de analizar, junto a dicho concepto, la evolución de la cultura.

Una similar comparación nos hace Huntington (1993) cuando nos proporciona un análisis cultural de la civilización occidental frente a la del Medio Oriente; busca interpretar desde una perspectiva totalizadora este contexto de globalización hegemónica del capitalismo mundial, lo único que une al continente occidental.

La cultura se manifiesta como sistema a través del conjunto de valores y creencias que comparten las personas que pertenecen a dicho sistema, así como por las múltiples formas en que se manifiestan dichos valores y creencias (Campos, 2014).

La visión moderna de cultura podemos encontrarla en la definición de Barfield, citando a Edward B. Tylor: "Cultura es ese todo complejo que incluye conocimiento, creencia, arte, moral, derecho, costumbre y cualesquiera otras capacidades y hábitos adquiridos por el hombre como miembro de una sociedad" (Barfield, 2000).

Es decir, que el ser humano desarrolla conocimiento cuando se relaciona con su entorno; cuando hace que el objeto se transforme en una propiedad que garantice transformar su entorno. Tanto la acción de tomar un objeto como la posibilidad de utilizarlo para el trabajo son dos acciones recíprocas, que son posibles con el raciocinio humano. Ese puente que hace posible dar nombre al objeto lo llamamos cultura; por eso es también conocimiento.

\section{La economía de Chiltiupán}

Los principales cultivos que se desarrollan dentro del municipio de Chiltiupán son: bálsamo, café, granos básicos, hortalizas y frutas. Dada la composición poblacional y el desarrollo productivo basado en su mayoría en agricultura, se ubica a Chiltiupán como municipio eminentemente rural (Recinos, 2008).

En la actualidad no existe un verdadero estímulo para el desarrollo del cultivo del bálsamo, debido, principalmente, a su baja rentabilidad en el país y a las dificultades de comercialización en el mercado internacional (Peñate, 1997). Entre las industrias manufactureras que se desarrollan en el municipio se encuentran la elaboración de productos lácteos (leche, crema y queso) y la explotación balsamera. Ambas industrias se producen de manera artesanal; son familiares y comúnmente se comercializan en el puerto de La Libertad y Santa Tecla (Peñate, 1997).

Chiltiupán cuenta con mucha participación ciudadana, ya que existen organizaciones de la sociedad civil, especialmente Adescos, que ocupan mecanismos de participación, como las asambleas comunitarias, para velar por la ejecución adecuada de proyectos y poner en evidencia los problemas de sus localidades.

Aun con este capital social, Chiltiupán es uno de los municipios con ingreso por hogar mensual más bajo del departamento de La Libertad, por lo que está considerado, como el cuarto con pobreza más alta (63,4\%), siendo el $41,9 \%$ de población catalogada en extrema pobreza. El ingreso per cápita mensual es de $\$ 42.60$, el segundo menor del departamento, lo que equivale a $\$ 1.42$ por persona diariamente.

Dentro de las dos grandes corrientes de ayuda económica que recibe el municipio se encuentran las remesas familiares, que corresponden a $\$ 42.105 .70$ mensuales, lo que se traduciría como un quinto de la contribución externa del departamento; y el ingreso del Fodes, que corresponde a $\$ 588.042$ para el año 2006. Cabe mencionar que el municipio no cuenta con una fuerte fuente de ingresos por impuestos ni de catastro.

Hay tres tipos de bosques en Chiltiupán: muy húmedo, húmedo subtropical y seco tropical. Existen áreas de bosques conformados por arboles de bálsamo, ceiba, mangle, papaturro, conacaste, morro, cedro, copinol, cortés 
negro, zopilocuayo, tambor, capulín de monte, níspero y roble. Los tipos de suelo que se encuentran son latasoles arcillo rojizos, alfisoles y litosales. (Alcaldía municipal de Chiltiupán, junio 2004).

\section{El proceso de producción del bálsamo, siembra y cultivo}

El bálsamo es un producto de calidad natural que los salvadoreños distinguen como medicinal. Sin embargo, las propiedades no se limitan a la parte curativa, sino que también en aspectos gastronómicos, aromáticos y cosméticos. Nuestros antepasados valoraron el uso del bálsamo como esa fuente curativa natural por excelencia y la tradición de su aplicación continua en nuestros días (Anliker Palomo, 1981).

Actualmente, la Cordillera del Bálsamo es uno de los lugares donde este árbol crece naturalmente y forma parte del paisaje cultural. En El Salvador es utilizado en la medicina tradicional y en la industria. "Aquí usamos el bálsamo para muchas cosas. Siempre tenemos guardadito un 'pucho', ya sea para los hongos de la piel, el catarro o para espantar los zancudos; y a veces mezclado con vinagre para la comida. Sin eso... a saber qué haríamos"7. Su resina es muy apreciada en Europa y Estados Unidos por sus propiedades medicinales, cosméticas, aromáticas y gastronómicas. Se le atribuye muchos beneficios a este importante cultivo en la actualidad (Anliker Palomo, 1981).

Los métodos de producción han implicado un proceso de aprendizaje cultural a través de la transferencia de la experiencia y el hecho de que los jóvenes son los que aprenden de los viejos, desde luego a través del lenguaje lleno de símbolos que reflejan conceptos.

Hoy día, los más jóvenes tienen cierta renuencia a implicarse en los procesos de producción. El tema no es solo económico, también tiene bases en el desarrollo de la política del Estado enfocada en un modelo de servicios y finanzas, menos en la producción y muchísimo menos en lo agrícola. "No me gusta treparme al bálsamo; queda uno con mucho olor", ${ }^{8}$ dice uno de los muchachos. Detrás de esa expresión hay una transformación cultural de la sociedad.
El proceso de extracción de la resina es descrito en una guía del Ministerio de Economía.

Lo primero que hay que hacer para extraer la savia del bálsamo es el proceso del quemado. Calientan el árbol con algo parecido a una antorcha y este, al sentir el fuego, va soltando la savia; es ahí donde rápidamente se colocan los trapos para recoger la savia. Luego de la quema, se espera un mes y 15 días para recoger los trapos (ES, 2008).

Las descripciones siguientes guían este proceso, con sus problemas y dificultades.

Cuando llega el momento, se suben al árbol, pues los trapos están colocados hasta en la parte más alta del bálsamo; y se recolectan uno por uno colocándolos en un saco. Luego de la recolección los trapos pasan a ser hervidos, pero para que suelten toda la savia que llevan pegada se necesita que el agua esté hirviendo, de no ser así no se extrae nada de bálsamo. En un principio el bálsamo es de un color amarillo claro, parecido a la miel, pero poco a poco se oscurece en la medida en que se va secando el agua (ES, 2008).

Desde luego, este proceso requiere de un manejo completo de las cualidades del árbol, para que sea capaz de soltar el bálsamo en el punto que se requiere para obtener una buena calidad.

Cuando los trapos están hirviendo en el barril, se siente un olor dulce parecido a la vainilla, pero con un toque de picante o caliente como la canela. Cuando los trapos están soltando la savia, se procede a sacarlos y a echarlos a un tipo de costal, al que se llama prensa, el cual tiene por dentro un enrejado estilo maya ciclón (ES, 2008).

De ahí el olor a bálsamo que se encuentra en toda la comunidad, por todos lados donde los balsameros están.

Allí se colocan los trapos y luego se pasa a la torsión de estos, se amarra el costal y se coloca en un palo especial, el cual, con un movimiento fuerte y rápido, hace que el enrejado que está dentro del costal exprima los trapos; y así va saliendo el bálsamo, este se observa como si fuera una miel aceitosa. Cuando todo el bálsamo ha salido de 
El impacto cultural del árbol de bálsamo en Chiltiupán, La

Libertad, El Salvador. Julio César Martínez-Rivera, Julio Blanco, Rodrigo Colorado, Óscar Guerra, Héctor Antonio, pp. 25-33.
Revista entorno, abril 2015, número 58.

ISSN: 2218-3345. los trapos, se encuentra en una gran olla y se procede a desaguar con un guacalito, ya que el agua queda en la superficie (ES, 2008).

Observar todos estos pasos en el contexto y tiempo real es una maravilla; la fuerza del balsamero para ejecutar los giros de la "torcedera"; la importancia que se da a la relación entre los balsameros y la recolección del bálsamo hasta la última gota forma parte de este rito de obtención del oloroso líquido.

Y por último, en el proceso de purificación se procede a hervir la savia que está en la olla, para que no tenga ni un residuo de agua. Es aquí donde el bálsamo obtiene su color característico, pues pasa de un café claro a uno muy oscuro, casi negro, al cual se le da el nombre de resina de bálsamo (ES, 2008).

Debemos preguntarnos si dentro de algunos pocos años, una generación, habrá o no nuevos productores de bálsamo y si el este se habrá reproducido (naturalmente o como cultivo) o no. Eso será si existe una real política de empleo y esta considera importante lo agroexportador para el sector campesino, entre esto el bálsamo.

Las condiciones que necesita el bálsamo para su crecimiento deben ser bosques de lluvia tropical, por lo tanto, el clima de esta zona es caluroso con lluvias torrenciales en época de invierno, que resultan ser momentos difíciles para los cultivadores y procesadores.

El crecimiento del bálsamo está acompañado por la presencia de cafetos y árboles frutales como mango, aguacate, julupe, matasano y especies maderables, como cinco, mulato, Brasil y camaroncillo, todos árboles que crecen junto con el bálsamo y que son fácilmente reconocibles por los habitantes de la cordillera.

De acuerdo con observaciones empíricas, se ha considerado que la edad óptima para el aprovechamiento del bálsamo oscila entre los 12 y 15 años. De lento crecimiento, este árbol puede ser productivo aún 30 años más y puede superar los 100 años, consecuentemente, con mucho cuido. "Hay que cuidarlos, limpiarlos, como que son niños chiquitos que van creciendo, para que den su aceite, ¿verdad?"9
El cuidado del bálsamo se refleja no solamente en el cuido del árbol per se, sino que también en el aspecto territorial natural que ocupa, así como en su vinculación con la transferencia cultural, que además debe luchar contra los medios de comunicación, las nuevas tecnologías y la migración.

Además, se ha estado llevando a cabo cultivos de tipo emergente de granos básicos, que representan más recursos económicos para los productores. Esta realidad afecta negativamente el bálsamo como producto generacional histórico de nuestro país, El Salvador. La ley estatal es necesaria para la protección de los árboles de bálsamo, tanto como patrimonio cultural como producto natural.

Las condiciones que favorecen la extracción del bálsamo se encuentran en su mejor momento en la estación seca, que comprende de los meses de octubre hasta abril. Cabe resaltar que quienes se dedican a la extracción de dicho cultivo en su mayoría son personas que también se dedican a otros tipos de agricultura y que durante los meses de lluvia procuran la producción de cosechas de maíz, frijol y otros.

En las épocas lluviosas, la productividad del bálsamo se reduce a dos terceras partes en comparación con la estación seca debido a que el pañal (trozo de trapo) que se usa para absorber la resina es lavado por la lluvia, llevándose consigo parte de la resina o aceite.

Las extracciones que se desarrollan actualmente son de tipo artesanal, bajo un aprendizaje de componente generacional, es decir, su proceso de extracción se ha modificado muy poco desde el período prehispánico.

Durante las extracciones y el uso de la tecnología primaria, la comunidad se llena del olor a bálsamo; y es imposible entenderla si este olor no existe. La población usa el bálsamo para distintas actividades, como base para oler mejor, como medicamento y como ahuyentador de los bichos de la nocturnidad. El bálsamo forma parte del paisaje cultural de la cordillera.

${ }^{9}$ Habitante de Chiltiupán. 


\section{Comercialización}

Los intentos de suprimir la subsistencia el bálsamo, usando productos alternativos sintéticos, no ha significado un verdadero riesgo para el mercado del bálsamo durante la década de los 1980.

En el análisis del mercado del bálsamo, es decir, la oferta y la demanda como perspectiva para un futuro, la calidad del bálsamo de El Salvador es al menos estable. Eso sucede debido que la calidad del bálsamo natural, ante el sintético alemán, es mejor.

Merece atención considerar las perspectivas de uso interno del bálsamo. La demanda del bálsamo es inelástica y no se cuenta con substitutos naturales o químicos (Fuentes, 1993).

El mercado internacional del bálsamo salvadoreño es amplio, en cuanto a número de países compradores. De acuerdo con un estudio realizado (Fuentes, 1993), es a los Estados Unidos, Alemania, Francia e Inglaterra adonde se destina, en promedio, el $98 \%$ de las exportaciones de dicho producto, siendo el primero el principal importador.

Un estudio en torno a la exportación del bálsamo menciona a los principales comercializadores internacionales y las dificultades que presentan, como se explica a continuación. Las cantidades exportadas varían, dependiendo del comportamiento de los precios y la demanda, siendo el mercado manejado principalmente por seis compañías exportadoras: Freund International; Liebes S.A. de C.V., Lasally Barrientos S.A de C.V.; J. Raúl Rivera S.A. de C.V. y J.C. Regalado Exportlmport. De los mencionados anteriormente solo cuatro se dedican a la actividad exportadora debido a la poca rentabilidad del negocio. El producto se vende en su estado natural, debido a que en El Salvador no existe una industria que procese y transforme la oleorresina, lo cual limita un aumento en el valor agregado de las transacciones del país. Los mayores compradores son Estados Unidos, Alemania y Francia (Peñate, 1997).

\section{Familias balsameras: una estructura de relaciones solidarias}

A través de las relaciones de solidaridad se expresa la unidad familiar, algo que en las familias campesinas salvadoreñas está bien marcado. La familia es la institución social básica y está compuesta por hombres y mujeres que tienen una relación de parentesco, más o menos permanente, con derechos y obligaciones, y socialmente reconocida juntamente con su prole.

La familia de un balsamero se distribuye las actividades de extracción y procesamiento. Los jóvenes -que generalmente son hombres-se suben a los árboles a veces hasta quince metros, aunque ocasionalmente alguna mujer que también lo hace. Las esposas, o compañeras de vida, en general se dedican a la tarea reproductiva de la familia, y ocasionalmente trabajan en el proceso de purificación del bálsamo. Otros miembros extraen la resina de los trapos a través de la torcedera, y otros envasan el producto.

Estaesuna distribución del trabajoque implica solidariamente y de forma cooperativa a la economía familiar. No es extraño, pues, que el bálsamo sea algo más que un cultivo medicinal; es la amalgama social de la comunidad.

Esta amalgama hoy presenta ciertas fisuras. Como hemos comentado, los más jóvenes van prefiriendo nuevos oficios; y la ciudad llama a los jóvenes que piensan que, a pesar de los riesgos que esta tiene, las oportunidades de lograr ciertos ingresos pueden ser más fáciles de lograr. Esto no siempre es así.

\section{Conclusiones}

- El bálsamo es un producto de raigambre histórica, de mucha importancia para entender la estructura social y económica de la cordillera. La permanencia histórica del bálsamo, en la cordillera, lo ha hecho ya formar parte del paisaje natural y de las costumbres y tradiciones. La cordillera no se concibe hoy día sin el bálsamo.

- La historia del bálsamo, como producto apetecible económicamente, se remonta a la transferencia de productos de la Colonia hacia España. Durante aquella época, los productos principales que los españoles buscaban en estas regiones no estaban relacionados con metales preciosos, como fue en el caso de México o de la cordillera de los Andes con los incas, sino con los productos agrícolas como el cacao y, desde luego, el bálsamo.

- Los balsameros son productores que no siempre son 
El impacto cultural del árbol de bálsamo en Chiltiupán, La

Libertad, El Salvador. Julio César Martínez-Rivera, Julio Blanco, Rodrigo Colorado, Óscar Guerra, Héctor Antonio, pp. 25-33.
Revista entorno, abril 2015, número 58.

ISSN: 2218-3345. poseedores de los bosques, sino que a veces son gente que alquila el bosque para explotarlo. En este sentido, la distribución de la tierra, a pesar de la reforma de su tenencia en el año 1980, continúa siendo desigual. Muchos balsameros alquilan los terrenos donde se encuentran los árboles de bálsamo para así lograr la extracción del aceite.

- El bálsamo, como producto, ha sido desplazado por productos sintéticos alemanes, perdiendo cierta importancia económica. Europa, con su revolución industrial y las necesidades de resolver la falta de productos importados por la vía de la sustitución con químicos, ha generado sustitutos del bálsamo, y por eso ha habido un desplazamiento del bálsamo natural. Sin embargo, aún sigue siendo más requerido el natural, pero el sintético es más disponible y barato.

- Las familias balsameras comparten el trabajo alrededor del bálsamo, y eso produce una amalgama familiar duradera. La distribución de las tareas de la familia es en dos sentidos: un trabajo productivo ligado a quienes trabajan en el campo, las torcederas y el envasado; y un trabajo relacionado con un esquema conservador de la distribución de actividades familiares, en el que se destaca el cuido de la casa y los niños.

- Las nuevas generaciones de campesinos de la cordillera en Chiltiupán tienen poco interés en convertirse en agricultores y procesadores del bálsamo; y cada vez existen menos interesados en el aprendizaje de la ocupación. Esto parece ser el precio que se debe pagar por un modelo de desarrollo económico desbalanceado y que favorece más al área financiera y de servicios que a la agroprocesadora.

- Los productores trabajan más fuerte que los comercializadores. Sin embargo, estos se llevan la ganancia. La falta de formación en el mercadeo, la cultura de la intermediación y el aprovechamiento del trabajo poco asociativo de los balsameros favorece que los comercializadores hacia extranjero sean personas ajenas al municipio.

\section{Reconocimientos}

Agradecemos a la comunidad de balsameros de Chiltiupán, a su cooperativa y sus habitantes en general. Particularmente a la municipalidad, que nos ha sabido brindar muchísimo apoyo y contactos.

\section{Referencias}

Alcaldía municipal de Chiltiupán (Junio, 2004). “Plan participativo de desarrollo e inversión municipal". Chiltiupán: Alcaldía municipal de Chiltiupán.

Alvarado, N. (1996). “Estudio antropologico y literario del elemento mitico del huasque y la siguanaba en la tradición oral de la comunidad del canton el ragadio, municipio de Chiltiupán, departamento de la Libertad". San Salvador: UES.

Angeloni, E. (2012). Annual editions: Anthropology 11/12. New York, USA: McGraw-Hill.

Anliker Palomo, P.E. (1981). La comercialización del bálsamo a través de cooperativas. San Salvador: UCA.

Barfield, T. (2000). Diccionario de Antropología. México: Siglo Veintiuno editores.

Bonte Pierre, I.M. (1997). Diccionario de Etnología y Antropología. Barcelona: AKAL.

Browning, D. (1975). El Salvador, la tierra y el hombre. San Salvador: Dirección de Publicaciones. Ministerio de Educación.

Campos, S. (15/10/2014). "La Cultura: La cultura como sistema". Obtenido de http://www.edutec.edu.sv/salonvirtual/course/view.php?id=60

Canales, H. (1982). Bálsamo de El Salvador. San Salvador: Dirección de Publicaciones.

Castaneda Langlius, H. (1999). Estudios Generales sobre la especie Myroxylon balsamun en El Salvador. San José: Instituto Tecnológico de Costa Rica.

Conrad, K. (1997). Antropología cultura. Madrid: McGraw-Hill.

ES, M. d. (31 de Dic. de 2008). Ficha UE 9 Exportación del bálsamo hacia la Unión Europea, San Salvador, El Salvador: Minec.

Etnografía, D. d. (Sf). Etnografía de El Salvador. San Salvador: Concultura. 
Fuentes, R.E. (1993). “El Bálsamo en El Salvador: una especie con potencial económico". Revista Forestal, 39-41.

Gobierno de El Salvador (5 de julio de 1939). Diario Oficial, 5 de julio de 1939, tomo 127. Decreto s/n. San Salvador, El Salvador: Imprenta oficial.

Gobierno de El Salvador (5 de julio de 1939). Diario Oficial, 5 de julio de 1939, tomo 127. Decreto s/n. San Salvador, El Salvador: Imprenta oficial.

GTZ. Deutsche Gesellschaft für Technische Zusammenarbeit. (S.f). "Sistematización del proceso de fomento de la cadena de bálsamo".

Hope Ponce, M. (2000). De abominable a zurriburrico: Diccionario en la ENAH, (buscar sincretismo, el diccionario no posee numeración en su páginas.

Huntington, S. (1993). "The clash of civilizations". Foreign affairs, 11-18.

Instituto Nacional del Café. (1988). Monografía del Café. San Salvador.

Lara-Martínez, R. (2007). Balsamera bajo la guerra fría. San Salvador: Universidad Don Bosco.

M.A., C. C. (1979). "Estudio económico y social de la producción y comercialización del bálsamo de El Salvador". San Salvador: UES.
Mark, B.P. (1993). Antropología Lectura. Lecturas.

Mercaba (9/9/2014). Obtenido de http://mercaba.org/ Rialp/E/estructuralismo_sociologia.htm

O, M. P. (2003). El Salvador Historia General. San Salvador: Editorial Nuevo Enfoque.

Peñate, A.C. (1997). Estrategia Competitiva para la Comercialización del Bálsamo. San Salvador, El Salvador: Universidad José Matías Delgado editores.

Recinos, L. e. (2008). "Prouesta estratégica de desarroIlo económico local a partir de los esfuerzos articulados entre los actores locales y características ecoturísticas del municipio de Chiltiupán". San Salvador: UCA.

Rojas, L. (2008). Elementos conceptuales y metodológicos de la investigación cualitativa. San José: Editorial UCR.

Rubenstein, S. (1963). El Ser y la Conciencia y el pensamiento y los caminos de su investigación. México: Editorial Grijalbo.

Salarrué (1974). Catleya luna. Cultura, 73-79.

Sandoval Casilimas, C. (1996). Investigación cualitativa. Bogotá: Instituto Colombiano para el fomento de la Educación Superior. 